\title{
Managing a High Specific Activity Iobenguane Therapy Clinic: From Operations to Reimbursement
}

Sophia R. O’Brien, $\mathrm{MD}^{1}$ and Daniel A. Pryma, $\mathrm{MD}^{2}$

${ }^{1}$ Divison of Nuclear Medicine, Department of Radiology, Hospital of the University of Pennsylvania, Philadelphia, PA, USA

${ }^{2}$ Division of Nuclear Medicine, Department of Radiology, Perelman School of Medicine at theUniversity of Pennsylvania, Philadelphia, PA USA

Disclosures: Daniel A. Pryma: Research Grant, Siemens AG; Research Grant, 511 Pharma; Research Consultant, 511 Pharma; Research Grant, Progenics Pharmaceuticals, Inc; Research Consultant, Progenics Pharmaceuticals, Inc; Research Consultant, Actinium Pharmaceuticals, Inc; Clinical Trial Funding, Nordic Nanovector ASA; Research Consultant, Ipsen.

Corresponding Author:Daniel A. Pryma, MD 3400 Spruce Street

Donner 1022

Philadelphia PA 19104

215-349-5272

215-349-5843

dpryma@pennmedicine.upenn.edu

First Author:

Sophia R. O’Brien, MD3400 Spruce St

1 Silverstein - Radiology AdministrationPhiladelphia PA, 19104

Phone: 215-349-5953

Fax: 215-243-2351

Sophia.Obrien@pennmedicine.upenn.edu

Sophia was a $4^{\text {th }}$-year radiology resident and an Integrated Nuclear Medicine Fellow in the 2020-2021 academic year

Manuscript Word Count (Including references): 2225

Running Title: Managing an I-131 MIBG Therapy Clinic(38 characters with spaces) 


\begin{abstract}
Metaiodobenzylguanidine (MIBG, iobenguane) is a guanethidine analogue that targets the norepinephrine transporter and, when radiolabeled with the beta-emitter I-131, has been used with varying protocols to treat neuroendocrine tumors, including pheochromocytoma/paraganglioma (PPGL) around the globe for more than 35 years. In 2018 a high specific activity formulation (Azedra®) became the first, and currently only, Food and Drug Administration approved drug to treat unresectable, locally advanced, or metastatic PPGL. This editorial will discuss the operational aspects of clinical implementation and use of HSA ${ }^{131}$ I- MIBG in advanced PPGL patients.
\end{abstract}




\section{INTRODUCTION}

Metaiodobenzylguanidine (MIBG, iobenguane) is a guanethidine analogue that targets the norepinephrine transporter and, when radiolabeled with the beta-emitter I-131, has been used with varying protocols to treat neuroendocrine tumors, including pheochromocytoma/paraganglioma (PPGL), neuroblastoma, and carcinoid, in adults and children around the globe for more than 35 years (1). In 2018 a high specific activity (HSA) formulation (Azedra®) became the first, and currently only, Food and Drug Administration (FDA) approved drug to treat unresectable, locally advanced, or metastatic PPGL (2). Multiple clinical trials are currently investigating other applications of ${ }^{131}$ I-MIBG such as in children with metastatic neuroblastoma and in combination with PRRT for patients with carcinoid tumor (3,4). We will likely see increasing FDA-approved and off-label use of ${ }^{131}$ I-MIBG in the coming years. This editorial will discuss the operational aspects of clinical implementation and use of HSA ${ }^{131} \mathrm{I}-\mathrm{MIBG}$ in advanced PPGL patients. In addition to reviewing the literature, this editorial is supported by our experience performing over 150 PPGL treatments over the last 10 years in both the inpatient and outpatient setting.

\section{PRE-TREATMENT EVALUTION AND TREATMENT OVERVIREW}

Since HSA ${ }^{131}$ I-MIBG is the only FDA approved treatment for advanced PPGL, all patients with advanced PPGL in whom systemic anticancer therapy is being considered should undergo diagnostic

${ }^{123}$ I-MIBG imaging and HSA ${ }^{131}$ I-MIBG therapy should be considered first line in those with MIBGavid disease. Systemic therapy is not considered in patients solely on the basis of metastatic disease, but reserved for patients with clear evidence of disease progression or who have disease symptoms not controlled with supportive treatment. For example, poorly controlled catecholamine-mediated symptoms (such as hypertension or anxiety) or disease-related pain can be an indication for therapy in 
the absence of objective progression.

Authorized user physicians evaluate prospective patients in clinic to ensure they meet criteria for treatment with ${ }^{131}$ I-MIBG, to explain the treatment protocol and goals of therapy, and to engage in shared decision making regarding if and when to move forward with treatment. Patients are counseled that therapy is intended to halt progression of their disease and/or decrease their symptoms, hopefully for many years, but is not a disease cure (5,6). Diagnostic I-123 MIBG scintigraphy is obtained to confirm MIBG-avid disease and to demonstrate the patient's baselinedisease burden.

In patients pursuing ${ }^{131}$ I-MIBG therapy, baseline bloodwork is obtained to confirm certain safety metrics are met, notably that platelets are $>80,000 / \mu \mathrm{L}$, absolute neutrophil count $>1,200 / \mu \mathrm{L}$, and eGFR >30. A negative pregnancy test is confirmed in women of childbearing potential and all patients are counseled to use effective contraception during treatment and for approximately 6 months after their final therapy. Recent baseline biochemical tumor markers (chromogranin A, catecholamines, metanephrines) and anatomic imaging are important for subsequent response assessment (noting that anatomic imaging may be unhelpful in patients with bone dominant disease). To protect patients' thyroid function, inorganic iodine is given starting the day before and for ten days after each treatment. For a complete discussion of pre-treatment patient preparation, please see the HSA ${ }^{131} \mathrm{I}-\mathrm{MIBG}$ prescribing information (2).

The recommended dosing regimen includes planar dosimetry with three anterior and posterior whole body scans done over 3-5 days after intravenous administration of about 185 MBq HSA ${ }^{131}$ I-MIBG. The maximum cumulative administered activity without exceeding organ limits is calculated. For onlabel indications there is no cost for the dosimetry dose. If a site does not have the expertise and/or software for dosimetry, third party services are available.

The recommended administered activity for therapy is $296 \mathrm{MBq} / \mathrm{kg}(8 \mathrm{mCi} / \mathrm{kg})$ up to a maximum of 
18.5 GBq $(500 \mathrm{mCi})$ in each of two treatment cycles given at least 90 days apart. For dosimetry revealing a maximum cumulative activity $<37 \mathrm{GBq}$, the prescribed activity for each cycle should be decreased equally. Most patients will require inpatient therapy; however, in patients with more indolent disease, lower administered activities can be given in an outpatient setting with reported efficacy. 74 $\mathrm{MBq} / \mathrm{kg}(2 \mathrm{mCi} / \mathrm{kg}) \times 4$ cycles at three month intervals is a common approach; $7.4 \mathrm{GBq}(200 \mathrm{mCi})$ per cycle has also been used.

In between cycles, bloodwork is obtained to ensure the absolute values and overall trends are safe before proceeding with the next therapy. Myelosuppression, specifically thrombocytopenia, is the most common toxicity of Azedra ${ }^{5}$. Recovery from nadir levels is usually seen by 4-6 weeks after treatment. Febrile neutropenia, Grade 4 thrombocytopenia $(<25,000 / \mu \mathrm{L})$, or neutropenia $(<500 / \mu \mathrm{L})$ lasting more than one week should prompt a $15 \%$ reduction in prescribed activity for subsequent treatment.

Post-treatment scintigraphic imaging is obtained about 5-7 days after therapy (or earlier on the day of hospital discharge for inpatients) to confirm successful radiotracer tumor targeting and which sometimes reveals sites of disease not well seen on the lower dose ${ }^{123}$ I-MIBG diagnostic imaging. Delayed post-treatment imaging, largely focused on anatomic imaging with CT or MR (ideally matching baseline imaging) with ${ }^{123}$ I-MIBG scintigraphy as an adjunct, is obtained 3 months or more after the completion of therapy. Assessing response to therapy is usually best done with anatomic imaging, biochemical tumor markers, and patient symptoms. A follow-up visit in the nuclear medicine clinic is scheduled to discuss the post-treatment imaging, bloodwork, symptoms, and next steps. Frequently the authorized user physician transfers primary oversight of the patient to his/her medical oncologist, endocrinologist or nephrologist, depending on local expertise. 


\section{SYSTEM INFRASTRUCTURE AND INPATIENT STAY}

Most HSA ${ }^{131}$ I-MIBG administrations will require an inpatient stay for radiation isolation. Patients will be given a private hospital room which is "wrapped" - the floor and surfaces covered with impervious materials for easy cleaning and disposal after the patient's stay.

Rolling shields may be required to minimize radiation exposure to adjacent rooms. Depending on room size, administered activity, and adjacent room occupancy (including above and below) shielding requirements can vary greatly. Therapy is infused intravenously by a trained nuclear medicine technologist or authorized user. Many methods for infusion have been described but a simple leadshielded syringe pump is felt to be the most straightforward method with least potential for contamination. The recommended infusion duration in adults is 30 minutes but as pharmacologic effects from ${ }^{131}$ I-MIBG have not been observed with the HSA preparation (in contrast to low specific

activity ${ }^{131}$ I-MIBG), more rapid infusions can be cautiously considered. To decrease exposures, staff should enter the patient's room as little as needed but can attend to all of a patient's medical needs. A Geiger Mueller counter with hand and foot monitoring attachments outside the patient room is very helpful to assess for potential contamination each time staff leave the room. Additionally, providing instant-read dosimeters to healthcare staff reassures that exposures are low and can spur immediate staffing changes in the unlikely event of higher exposures. We have found that with these measures in place, nursing care for our admitted patients is very straightforward and often welcomed by the nurses, as our patients tend to be far less sick than the typical oncology inpatient.

Nausea and vomiting are almost ubiquitous with high dose therapy and scheduled antiemetics are recommended for all patients. Intravenous fluids are also recommended for all patients to minimize nausea and improve unbound radiotracer clearance. Intravenous fluids are continued for as long as the patient can tolerate, limited either by patient preference or by signs or symptoms of volume overload. 
Since the majority of ${ }^{131} \mathrm{I}-\mathrm{MIBG}$ is excreted intact in the urine, bladder catheterization is recommended for young children and incontinent adults during hospitalization. Continent patients are encouraged to empty their bladder frequently to decrease bladder exposure. To reduce potential contamination, patients should sit when urinating, double flush the toilet, and carefully wash their hands.

Damaged and dying neuroendocrine cells can paroxysmally release large amounts of catecholamines, leading to blood pressure lability or hypertensive crisis. While most common within two days of treatment, lability can persist for weeks and titration of antihypertensives may be needed (in many patients the optimal antihypertensive regimen will decrease after therapy) ${ }^{2}$. Additionally, in patients with catecholamine-induced hyperglycemia, hypoglycemic drugs may require decreasing doses after therapy.

Radiation safety personnel will perform daily assessments of inpatients' radiation levels. In most of the United States, patients can be discharged when the exposure rate at 1 meter from the patient is less than $70 \mu \mathrm{Sv} / \mathrm{hr}$ (7 mrem/hr). Hospital stay for radiation isolation typically lasts 3-5 days after an $18.5 \mathrm{GBq}$ administration but varies based on multiple factors including total administered activity, overall tumor burden, organ function, patient's home living situation and local regulations. The kinetics of MIBG are very similar to those of sodium iodide, so radiation precautions and outpatient dose limits used for thyroid cancer patients treated with sodium I-131 can also be applied to PPGL patients treated with ${ }^{131}$ IMIBG.

\section{MULTIDISCIPLINARY STAFF}

As for other therapeutic and diagnostic nuclear medicine agents, the handling and administration of HSA ${ }^{131}$ I-MIBG requires a well-trained multidisciplinary staff. A licensed authorized user as defined 
by the United States Nuclear Regulatory Commission (NRC) is responsible for the overall safe handling of radiopharmaceuticals, including MIBG. Nuclear medicine technologists accept, store, and handle MIBG according to standard NRC and agreement state operating procedures.

Health/medical physicists collaborate with authorized users to quantify safe levels of administered activities for each patient. Nurses, radiation safety officers, and environmental services employees work in patient- and non-patient-facing roles to ensure the safety of patients, their families, and hospital staff.

Institutions which currently administer other nuclear therapies likely have this multidisciplinary staff in place and typically no new resources are needed to initiate an HSA ${ }^{131}$ I-MIBG therapy program. If needed, training given to nuclear medicine staff can easily be adapted to nursing and other healthcare staff. Periodic in-services and written standard operating procedures are recommended since PPGL patients are rare. Easy accessibility to a health physicist is recommended for any staff questions or concerns.

\section{PURCHASING AND REIMBURSEMENT}

Each HSA ${ }^{131}$ I-MIBG patient will require a dosimetry dose and, usually, multiple therapy doses. The HSA ${ }^{131}$ I-MIBG manufacturer provides a list of dates on which doses are available. As above, there is no charge for the dosimetric dose for PPGL patients treated according to the approved indication, though the site will want to bill for the imaging acquisition. Billing for dosimetry calculations themselves is potentially feasible; guidelines are currently being created by an SNMMI Dosimetry Task Force and will be published separately. It can be helpful to provisionally place an order for a patient's expected treatment dose at the time that the dosimetrydose is ordered. 
Most insurance providers have national coverage decisions for HSA ${ }^{131} \mathrm{I}-\mathrm{MIBG}$ therapy; however, given the rarity of the disease, it may not be included in individual center contracts. Pre-authorization is required. Additionally, working with a site's billing staff to create single patient agreements with the insurance provider is recommended as a best practice. Given the rarity of the disease, this is a straightforward process with most payers. While standard coverage policies/contracts will provide adequate reimbursement for most outpatient therapies, single patient agreements help to ensure that full drug acquisition cost is added to the standard inpatientreimbursement.

The "Azedra Service Connection" is a manufacturer program designed to help providers order therapy and to help patients and providers navigate treatment logistics and payment. Financial assistance is available for eligible uninsured patients and may cover the entirety of the treatment costs. Because HSA

${ }^{131}$ I-MIBG can be ordered at a per mCi cost, prescribing a lower outpatient administered activity is easily scalable. CMS has granted a temporary new technology add on payment to increase the reimbursement for inpatient Azedra therapy for Medicare beneficiaries. With pre-authorization and single patient agreements, we have found providing this unique treatment to this ultra-orphan patient population to be economically viable.

\section{CONCLUSION}

An ${ }^{131}$ I-MIBG therapy clinic is easily managed from both an operational and financial aspect and serves an important role in the multidisciplinary care for PPGL patients and potentially other neuroendocrine tumor patient populations. 


\section{DISCLOSURES}

Daniel A. Pryma discloses research grants from Siemens AG, 511 Pharma, and Progenics Pharmaceuticals, Inc; research consultant positions with 511 Pharma, Progenics Pharmaceuticals, Inc, Ipsen, and Actinium Pharmaceuticals, Inc; and Clinical Trial Funding from Nordic Nanovector ASA. No other potential conflicts of interest relevant to this article exist. 


\section{References}

1. Tobes MC, Jaques S, Wieland DM, Sisson JC. Effect of uptake-one inhibitors on the uptake of norepinephrine and Metaiodobenzylguanidine. J Nucl Med. 1985;26:897-907.

2. AZEDRA [package insert]. New York, NY: Progenics Pharmaceuticals Inc; 2018.

3. New approaches to neuroblastoma therapy consortium. 131I-MIBG alone vs. 131I-MIBG with Vincristine and Irinotecan VS131I-MIBG with Vorinistat (N2011-01).

4. Bushnell DL, Bodeker KL, O'dorisio TM, et al. Addition of 131 I-MIBG to PRRT (90YDOTATOC) for personalized treatment of selected patients with neuroendocrine tumors. J Nucl Med. Published online January 30, 2021. doi:10.2967/jnumed.120.254987.

5. Pryma DA, Chin BB, Noto RB, et al. Efficacy and safety of high-specific activity 131I- MIBG therapy in patients with advanced pheochromocytoma or paraganglioma. J Nucl Med. 2019;60:623-630.

6. AZEDRA [patient brochure]. New York, NY: Progenics Pharmaceuticals In; 2019. 